\title{
A very massive runaway star from Cygnus OB2^
}

\author{
F. Comerón ${ }^{1}$ and A. Pasquali ${ }^{2}$ \\ 1 ESO, Karl-Schwarzschild-Str. 2, 85748 Garching bei München, Germany \\ e-mail: fcomeron@eso.org \\ 2 Max-Planck Institut für Astronomie, Königstuhl 17, 69117 Heidelberg, Germany \\ e-mail: pasquali@mpia.de
}

Received 15 February 2007 / Accepted 3 April 2007

\begin{abstract}
Aims. We investigate the star $\mathrm{BD}+43^{\circ} 3654$ and the possibility that it may have originated in the massive $\mathrm{OB}$ association Cygnus $\mathrm{OB} 2$. Methods. We present new spectroscopic observations allowing a reliable spectral classification of the star, and discuss existing MSX observations of its associated bow shock and astrometric information not previously studied.

Results. Our observations reveal that $\mathrm{BD}+43^{\circ} 3654$ is a very early and luminous star of spectral type O4If, with an estimated mass of $(70 \pm 15) M_{\odot}$ and an age of $\sim 1.6 \mathrm{Myr}$. The high spatial resolution of the MSX observations allows us to determine its direction of motion in the plane of the sky by means of the symmetry axis of the well-defined bow shock, which matches well the orientation expected from the proper motion. Tracing back its path across the sky we find that BD $+43^{\circ} 3654$ was located near the central, densest region of Cygnus $\mathrm{OB} 2$ at a time in the past similar to its estimated age.

Conclusions. $\mathrm{BD}+43^{\circ} 3654$ turns out to be one of the three most massive runaway stars known, and it most likely formed in the central region of Cygnus OB2. A runaway formation mechanism by means of dynamical ejection is consistent with our results.
\end{abstract}

Key words. stars: early-type - stars: kinematics - stars: individual: BD+43 3654 - Galaxy: open clusters and associations: individual: Cygnus OB2

\section{Introduction}

Current theories for the formation of massive stars stress the importance of the dense cluster environment in which most, if not all, form (Bonnell et al. 2007). Dynamical interactions at the centers of massive star forming regions lead to captures forming binary systems, ejections, mass segregation, and possibly coalescence. A remarkable byproduct of the dynamical interactions in dense clusters of massive stars is the relatively large abundance of runaway O-type stars, which amount to almost $\sim 10 \%$ of the known O-type stars in the solar vicinity (see Maíz-Apellániz et al. 2004 for a recent census). Runaway stars, characterized by their high spatial velocities, can form either by dynamical ejection from a dense cluster (Poveda et al. 1967; Leonard \& Duncan 1988, 1990) or by the explosion as supernova of a member of a close massive binary (Blaauw 1961; van Rensbergen et al. 1996; de Donder et al. 1997). Evidence for both mechanisms exists (Hoogerwerf et al. 2000, 2001), and both are a consequence of the special conditions in which massive star formation takes place. On the one hand, the high stellar density of the parental cluster facilitates the dynamical ejection scenario. On the other hand, the supernova scenario is favored by the high frequency of binaries with high mass ratios among massive stars (Garmany et al. 1982; Preibisch et al. 1999), which

* Based on observations collected at the Centro Astronómico Hispano-Alemán (CAHA) at Calar Alto, operated jointly by the MaxPlanck Institut für Astronomie and the Instituto de Astrofísica de Andalucía (CSIC). may be a consequence of dynamical capture followed by accretion and orbital evolution (Bate et al. 2003).

Cygnus OB2, the most massive OB association of the solar neighbourhood (Knödlseder 2000, 2003; Comerón et al. 2002, and references therein), should be the source of numerous runaway stars given its rich content in massive stars, which includes the massive multiple system Cyg OB2 8 near its center. Unfortunately, few studies have addressed its possible runaway population, with the exception of the recent radial velocity survey of Kiminki et al. (2007) in which no runaway candidate has been identified. Comerón et al. (1994, 1998) pointed out the existence of large-scale kinematical peculiarities in the Cygnus region, most likely related to the presence of Cygnus OB2, as shown by Hipparcos proper motions. Although they interpreted their results in terms of triggered star formation (Elmegreen 1998), at least some of the stars that they identified as moving away from Cygnus OB2 might be actual runaways formed by either of the two mechanisms listed above.

In this paper we report the identification of a very high mass runaway star, $\mathrm{BD}+43^{\circ} 3654$, very probably ejected from Cygnus OB2. The star had been identified as a likely runaway by van Buren \& McCray (1988) based on the existence of an apparent bow shock in IRAS images, caused by the interaction of its stellar wind with the local interstellar medium. Here we present the first spectroscopic observations of the star, which show it to be a very early Of-type supergiant. We also present proper motion data and higher resolution MSX images leading to a more detailed analysis, which strongly supports an origin at the core of Cygnus OB2. 


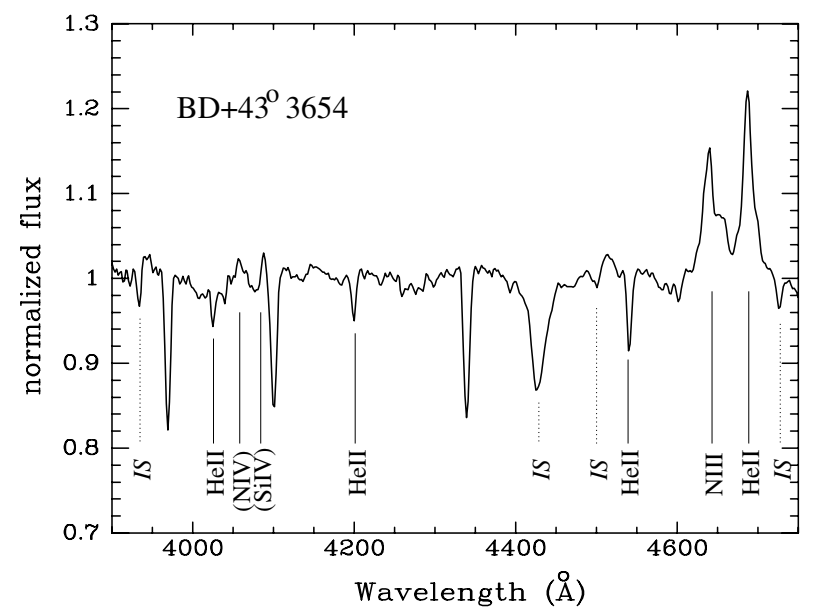

Fig. 1. Spectrum of BD $+43^{\circ} 3654$ showing the main absorption lines used for spectral classification and the prominent emission of NIII and HeII. The prominent, unlabeled features are $\mathrm{H} \gamma, \mathrm{H} \delta$, and $\mathrm{H} \epsilon$. Interstellar absorption features are indicated by dotted lines. The locations where one might expect to detect NIV and SiIV transitions are also indicated.

\section{Observations}

The spectrum presented here was obtained in the course of a project aimed at producing spectral classifications of previously unknown, photometrically selected new OB stars in the surroundings of Cygnus OB2. The photometry in the $B R J H K_{\mathrm{S}}$ bands was taken from the Naval Observatory Merged Astrometric Dataset (NOMAD) catalog (Zacharias et al. 2004), which combines astrometry and photometry from the Hipparcos, Tycho-2, UCAC2, USNO-B1.0, and 2MASS catalogs. The spectroscopic observations were carried out with the $2.2 \mathrm{~m}$ telescope at the German-Spanish Astronomical Center on Calar Alto (Spain) using the CAFOS visible imager and spectrograph. A $1^{\prime \prime} 5$ slit combined with the B-100 grism, providing a resolution $\lambda / \Delta \lambda=800$ in the blue part of the visible spectrum, were used. The exposure time was $900 \mathrm{~s}$. The spectrum was reduced, extracted, and wavelength calibrated using standard IRAF ${ }^{1}$ tasks in the ONEDSPEC package, and it was ratioed by a sixth-degree polynomial fit to the continuum in order to remove the steep slope due to the strong extinction towards the star.

\section{Results}

\subsection{Stellar classification, properties, and kinematics}

Although the identification of $\mathrm{BD}+43^{\circ} 3654$ as a likely runaway star dates back to van Buren \& McCray (1988), no spectral classification is available in that work. Subsequent papers by van Buren et al. (1995) and Noriega-Crespo et al. (1997) refer to the star as a unspecified B-type but do not report dedicated observations, and no other spectroscopic classification appears to be available in the literature apart from a generic classification as "OB reddened" in the LS catalog (Hardorp et al. 1964). The spectrum presented here is thus the first one allowing an accurate spectral classification of $\mathrm{BD}+43^{\circ} 3654$ and an estimate of its physical parameters.

The most obvious spectroscopic feature of BD $+43^{\circ} 3654$ is the presence of intense emission in the NIII and HeII lines, and

${ }^{1}$ IRAF is distributed by NOAO, which is operated by the Association of Universities for Research in Astronomy, Inc., under contract to the National Science Foundation. possibly also in NIV and SiIV, clearly indicating that it is an Of star. HeII lines are also prominent in absorption, and together with the absence of HeI lines indicates a spectral type earlier than O5. Absorption bands due to interstellar absorption, CaII and diffuse interstellar bands, are also strong due to the high extinction towards the star. Based on comparison with the atlas of Walborn \& Kirkpatrick (1990), we classify the star as O4If. Using intrinsic colors of early-type stars from Tokunaga (2000) and the 2MASS $H K_{\mathrm{S}}$ photometry from the NOMAD catalog, we estimate a $K$-band extinction $A_{K}=0.57 \mathrm{mag}$.

A summary of previous distance determinations to Cygnus OB2 has been presented by Hanson (2003). Based on her results, we adopt her favored distance modulus $D M=10.8$ corresponding to a distance of $1450 \mathrm{pc}$, with an estimated uncertainty of \pm 0.4 based on the results of previous determinations summarized in that work. Assuming that BD+43 3654 is approximately at the same distance from the Sun as Cygnus OB2, we derive its absolute magnitude as

$M_{V}=K_{\mathrm{S}}-A_{K}+\left(V-K_{\mathrm{S}}\right)_{0}-D M=-6.3 \pm 0.5$

where the 0.5 mag uncertainty includes as the dominant source the quoted uncertainty in the distance modulus and the contribution of error in the derivation of the extinction. We estimate the latter to be 0.2 mag based on the quality of the fit of a reddened O4-type spectral energy distribution to the measured $B R J H K_{\mathrm{S}}$ photometry. The contribution of the errors in the broad-band photometry is negligible as compared to those other two sources.

Different calibrations of the stellar parameters of O stars can be found in the literature to estimate the mass and the age of $\mathrm{BD}+43^{\circ} 3654$. These calibrations are based on a different treatment of the stellar model atmospheres, depending on whether non-LTE conditions, line-blanketing effects and stellar winds are taken into account. For an O4 supergiant in the Milky Way (i.e. of solar metallicity), Martins et al. (2005) provide an effective temperature $T_{\text {eff }}=40702 \mathrm{~K}$; Repolust et al. (2004) estimate a colder $T_{\text {eff }}=39000 \mathrm{~K}$, while Vacca et al. (1996) give $T_{\text {eff }}=47690 \mathrm{~K}$. We have adopted the average of those three calibrations, $42464 \mathrm{~K}$, as the temperature for $\mathrm{BD}+43^{\circ} 3654$, considering as the uncertainty the range of temperatures spanned by those models. This uncertainty is larger than the temperature difference between the subtypes $\mathrm{O} 4$ and $\mathrm{O} 5$, and between types $\mathrm{O} 4 \mathrm{I}$ and $\mathrm{O} 4 \mathrm{~V}$, for any given calibration (see e.g. Martins et al. 2005). The same is true for the effects of metallicity, which are hardly noticeable even when metal abundances change by a factor of 10 . This is clearly shown in Fig. 15 of Heap et al. (2006), where the temperature-spectral type relationships from different calibrations involving both galactic and Small Magellanic Cloud O stars are compared. Therefore, plausible uncertainties in either our spectral classification or in our assumption of solar metallicity for $\mathrm{BD}+43^{\circ} 3654$ do not significantly alter the size of the error bars in Fig. 2. The absolute magnitudes $M_{\mathrm{V}}$ obtained by all three models are very similar, with an average of $M_{\mathrm{V}}=-6.36$ and individual determinations deviating by less than $0.05 \mathrm{mag}$ from that value. This is remarkably close to the value that we obtain from the photometry of $\mathrm{BD}+43^{\circ} 3654$ and the assumption that its distance modulus is the same obtained by Hanson (2003) for Cygnus OB2, thus supporting our choice of that distance for the star.

The position of $\mathrm{BD}+43^{\circ} 3654$ on the Herzsprung-Russell (HR) diagram is shown in Fig. 2, together with the isochrones computed by Meynet et al. (1994) for solar metallicity and with enhanced stellar mass loss. These evolutionary tracks are preferable because they better reproduce the low-luminosity observed for some Wolf-Rayet stars, the surface chemical composition of 


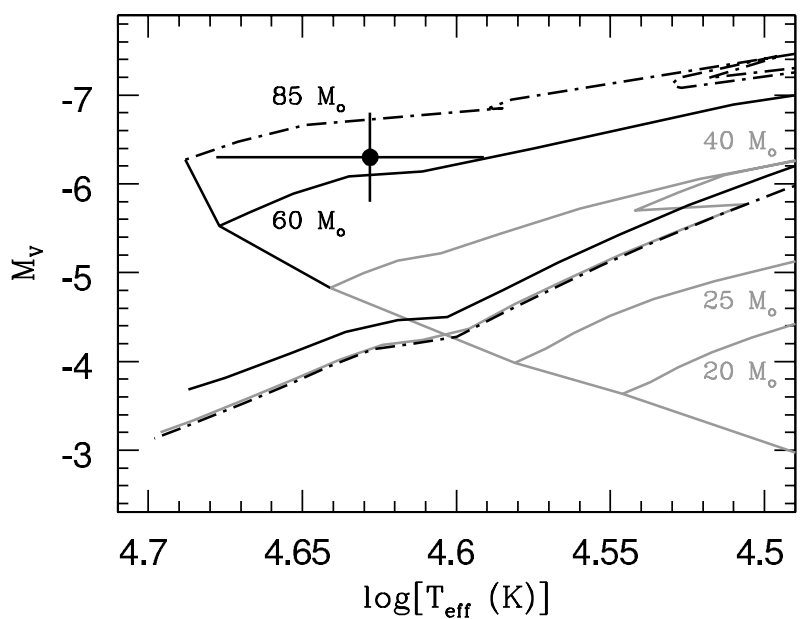

Fig. 2. Comparison between the position of BD $+43^{\circ} 3654$ and the evolutionary tracks for very massive stars of Meynet et al. (1994).

WC and WO stars and the ratio of blue to red supergiants in the star clusters of the Magellanic Clouds. The isochrones plotted in Fig. 2 refer to a stellar mass of the progenitor on the main sequence of $M_{i}=20,25,40 M_{\odot}$ (in grey) and $M_{i}=60,85 M_{\odot}$ (in black). The comparison between the observed properties of $\mathrm{BD}+43^{\circ} 3654$ and the isochrones allows us to estimate an initial mass $M_{i} \simeq(70 \pm 15) M_{\odot}$ and an approximate age of $1.6 \mathrm{Myr}$. The isochrones do not take into account stellar rotation, which many studies in the past decade have found to greatly affect mixing and mass loss, and to be an important ingredient for stellar evolution (Meynet \& Maeder 1997; Langer et al. 1998; Heger \& Langer 2000; Meynet \& Maeder 2000, and references therein). As shown by Meynet \& Maeder (2000), for an initial rotational velocity of $200-300 \mathrm{~km} \mathrm{~s}^{-1}$ and solar metallicity isochrones become brighter by a few tenths of a magnitude and the lifetime in the H-burning phase increases by $20-30 \%$. Given the observational errors on $\mathrm{BD}+43^{\circ} 3654$, these changes do not significantly affect our estimates of the initial mass and age of the star.

Proper motions for $\mathrm{BD}+43^{\circ} 3654$ are available from the NOMAD catalog, based on measurements by the Hipparcos satellite in the Tycho catalog further refined with previous ground-based observations. The values listed are $\mu_{\alpha} \cos \delta=$


corresponding values expressed in galactic coordinates, which are more convenient to derive the spatial velocity of the star with respect to its local interstellar medium, are $\mu_{l} \cos b=$

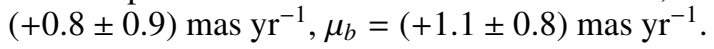

Assuming that the local interestellar medium in the surroundings of $\mathrm{BD}+43^{\circ} 3654$ moves in a circular orbit around the galactic center, its proper motion $\left(\mu_{l} \cos b\right)_{0},\left(\mu_{b}\right)_{0}$ can be described by the first-order approximation to the local galactic velocity field; see e.g. Scheffler \& Elsässer (1987):

$$
\begin{aligned}
\left(\mu_{l} \cos b\right)_{0}= & 0.211[A \cos 2 l \cos b+B \cos b \\
& \left.+\frac{U}{D} \sin l-\frac{V}{D} \cos l\right]
\end{aligned}
$$

$$
\begin{aligned}
\left(\mu_{b}\right)_{0}= & 0.211[-A \sin 2 l \sin b \cos b \\
& \left.+\frac{U}{D} \cos l \sin b+\frac{V}{D} \sin l \sin b-\frac{W}{D} \cos b\right]
\end{aligned}
$$

where $A$ and $B$ are the Oort constants in units of $\mathrm{km} \mathrm{s}^{-1} \mathrm{kpc}^{-1}$; $U, V$, and $W$ are the components of the solar peculiar motion in the directions toward the galactic center, the direction of circular galactic rotation, and the North galactic pole respectively, in $\mathrm{km} \mathrm{s}^{-1}$, and $D$ is the distance to the Sun in kpc. We have adopted $A=-B=12.5 \mathrm{~km} \mathrm{~s}^{-1} \mathrm{kpc}^{-1}$, corresponding to a flat rotation curve with an angular velocity of $25 \mathrm{~km} \mathrm{~s}^{-1} \mathrm{kpc}^{-1}$ and $(U, V, W)=(7,14,7) \mathrm{km} \mathrm{s}^{-1}$. The proper motion of $\mathrm{BD}+43^{\circ} 3654$ with respect to its local interstellar medium is then

$$
\begin{aligned}
\Delta \mu_{l} \cos b & =\mu_{l} \cos b-\left(\mu_{l} \cos b\right)_{0}=(5.3 \pm 1.1) \mathrm{mas} \mathrm{yr}^{-1} \\
\Delta \mu_{b} & =\mu_{b}-\left(\mu_{b}\right)_{0}=(2.0 \pm 0.9) \mathrm{mas} \mathrm{yr}^{-1}
\end{aligned}
$$

where the uncertainty allows for an error of $2 \mathrm{~km} \mathrm{~s}^{-1} \mathrm{kpc}^{-1}$ in each of $A, B$, and $2 \mathrm{~km} \mathrm{~s}^{-1}$ in each of $U, V$, and $W$. The position angle $\theta$ of the residual proper motion with respect to the North galactic pole, counted as positive in the direction of increasing galactic latitude, is then

$$
\theta=\tan ^{-1} \frac{\Delta \mu_{l} \cos b}{\Delta \mu_{b}}=69^{\circ} 3 \pm 9^{\circ} 4 .
$$

The component of the spatial velocity on the plane of the sky that we derive from the residual proper motion at the adopted distance of $1450 \mathrm{pc}$ is $(39.8 \pm 9.8) \mathrm{km} \mathrm{s}^{-1}$, which is several times the sound speed in a warm neutral interstellar medium at a temperature of $\sim 8000 \mathrm{~K}$, as expected from the fact that a clear bow shock is observed ahead of the star in the direction of its motion.

\subsection{The bow shock}

The original identification of a possible bow shock associated with $\mathrm{BD}+43^{\circ} 3654$ was reported by van Buren \& McCray (1988) based on $60 \mu \mathrm{m}$ IRAS maps, and further details were given by van Buren et al. (1995) and Noriega-Crespo et al. (1997). While indeed suggestive of a bow shock, the resolution of the IRAS $60 \mu \mathrm{m}$ images presented by van Buren et al. (1995) is not sufficient to accurately determine the shape of the bow shock and its position with respect to the star.

Much improved images of the region around BD+43 3654 have been provided by the Midcourse Space Experiment (MSX) satellite (Price et al. 2001). The BD+43 3654 bow shock appears in them as a neat, well defined arc-shaped nebula in the $D$ (13.5-15.9 $\mu \mathrm{m}$; see Fig. 3) and $E(18.2-25.1 \mu \mathrm{m})$ bands, and is absent in the $A(6.8-10.8 \mu \mathrm{m})$ and $C(11.1-13.2 \mu \mathrm{m})$ bands. The position of the apsis of the bow shock with respect to $\mathrm{BD}+43^{\circ} 3654$ can be well determined from those images, being located at 3.4 arcmin from the star in a direction that forms an angle of $62^{\circ} 5 \pm 10^{\circ}$ with the direction towards the north galactic pole. This position angle is in very good agreement with the position angle of the residual velocity vector of the star (Eq. (4)).

The position of the bow shock with respect to the star allows us to estimate the density of the interstellar medium through which $\mathrm{BD}+43^{\circ} 3654$ is moving. The apsis of the bow shock is approximately located at the stagnation radius, which is the distance from the star where the ram pressure of the interstellar gas equals that of the stellar wind, given by (e.g. Wilkin 1996):

$R_{0}=\sqrt{\frac{\dot{M}_{\mathrm{w}} v_{\mathrm{w}}}{4 \pi \rho_{\mathrm{a}} v_{*}^{2}}}$

where $\dot{M}_{\mathrm{w}}$ and $v_{\mathrm{w}}$ are respectively the mass loss rate and terminal wind velocity of the star, $\rho_{\mathrm{a}}$ is the ambient gas density, and $v_{*}$ is the spatial velocity of the star. The distance given in Eq. (5) assumes that the bow shock is bound by shock fronts on both 


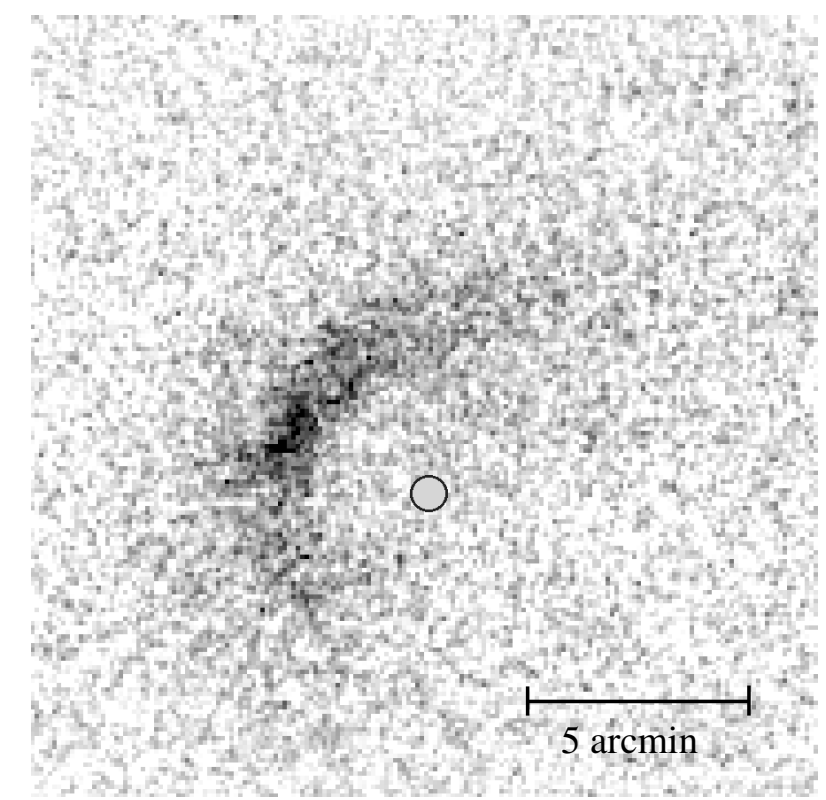

Fig. 3. Image obtained in the Midcourse Space Experiment (MSX) galactic plane survey in the $D$ medium-infrared band $(13.5 \mu \mathrm{m}-15.9 \mu \mathrm{m})$. The position of $\mathrm{BD}+43^{\circ} 3654$ is marked with a grey circle. The galactic North is up and the direction of growing galactic longitude to the left.

sides. In reality, the non-zero cooling time of the shocked stellar wind builds up a thick layer of low-density, high-temperature gas between the reverse shock on the stellar wind and the bow shock. The existence of this thick layer moves the position of the apsis of bow shock to a greater distance from the star than that given by Eq. (5). This expression actually gives the position of the reverse shock ahead of the star, as shown in numerical simulations by Comerón \& Kaper (1998), and the actual position of the bow shock is normally $\sim 1.5 R_{0}$, the precise distance depending on the quantities entering the right-hand side of Eq. (5) and the cooling curve of the stellar wind gas. Concerning the stellar wind, we have adopted $\dot{M}=10^{-5} M_{\odot} \mathrm{yr}^{-1}$ and $v_{*}=2300 \mathrm{~km} \mathrm{~s}^{-1}$ as typical values derived by Markova et al. (2004) and Repolust et al. (2004) for the O4I stars in their samples. Finally, we use $v_{*}=39.8 \mathrm{~km} \mathrm{~s}^{-1}$ as derived in the previous section, assuming for simplicity that most of the velocity of $\mathrm{BD}+43^{\circ} 3654$ is on the plane of the sky and that there are no projection effects on the position of the bow shock. Introducing these values in Eq. (5), we obtain a number density of the local interstellar gas $n_{\mathrm{H}} \simeq 6 \mathrm{~cm}^{-3}$. This is only a rough estimate of the density, mainly due to the large uncertainties in the values adopted for the different quantities intervening in Eq. (5) and the assumption that the residual motion of the star is in the plane of the sky. In particular, we note that Bouret et al. (2005) find mass loss rates smaller by a factor of $\sim 3$ for the galactic O4If+ star HD190429A when taking into account wind clumping with respect to the homogeneous wind case, which may imply an overestimate of $n_{\mathrm{H}}$ by a similar factor due to our adopted values. The estimated density clearly indicates that the star is moving in a tenuous medium whose density matches well that typical of the warm HI gas in the vicinity of the galactic midplane (e.g. Dickey \& Lockman 1990).

\section{Discussion: the origin of $B D+43^{\circ} 3654$}

The spectral type and estimated mass of $\mathrm{BD}+43^{\circ} 3654$ places it among the three most massive runaway stars known to date. The only other two comparable stars are $\zeta$ Pup and $\lambda$ Cep (spectral types O4I(n)f and O6I(n)fp, respectively; Maíz Apellániz 2004), whose masses $\left(65-70 M_{\odot}\right.$, as estimated by Hoogerwerf et al. 2001 from evolutionary models by Vanbeveren et al. 1998) are similar to the one that we estimate for $\mathrm{BD}+43^{\circ} 3654$.

Although currently placed near the boundary separating Cygnus OB1 and OB9 (to the extent that this boundary may be real; see Schneider et al. 2007), the proper motion of $\mathrm{BD}+43^{\circ} 3654$ points away from the core of Cygnus OB2, which is approximately marked by the location of the multiple system of O stars Cyg OB2 8A-D. Other early O-type stars, most notably Cyg OB2 22A (O3If*), Cyg OB2 22B (O6V((f))), and Cyg OB2 9 (O5If+), are also within few arcminutes of that location. The position angle of $\mathrm{BD}+43^{\circ} 3654$ with respect to this system is $58^{\circ} 84$, very similar to the position angle of its residual proper motion vector (Sect. 3.1) and of the axis of the bow shock (Sect. 3.2). In view of the high density of very massive OB stars found in the central regions of Cygnus OB2 (Massey \& Thompson 1991), we thus consider as a very likely possibility that $\mathrm{BD}+43^{\circ} 3654$ was formed there and subsequently expelled.

Assuming that $\mathrm{BD}+43^{\circ} 3654$ was born in the close vicinity of Cyg OB2 8, to which it is currently separated by an angular distance $\delta=2^{\circ} 67$, the travel time to its current position is $\tau=$ $\delta / \sqrt{\left(\Delta \mu_{l} \cos b\right)^{2}+\left(\Delta \mu_{b}\right)^{2}}=1.7 \pm 0.4 \mathrm{Myr}$, which is close to the age of the star inferred from the evolutionary tracks and the position in the H-R diagram (Sect. 3.1). The coincidence between the age and the travel time supports dynamical ejection early in its life as the cause of its runaway velocity, since there would have been no time for a hypothetical massive companion to evolve, go through different mass transfer episodes (Vanbeveren et al. 1998) and then explode as a supernova. The spatial velocities of the other two massive runaways noted above are probably higher, unless the radial velocity of $\mathrm{BD}+43^{\circ} 3654$ exceeds the projected velocity on the plane of the sky: Hoogerwerf et al. (2001) measure a velocity of $62.4 \mathrm{~km} \mathrm{~s}^{-1}$ for $\zeta$ Pup, and 74.0 for $\lambda$ Cep. High spatial velocities may be the signature of an origin by supernova ejection, since high ejection velocities by dynamical interaction become increasingly unlikely as the mass of the ejected star increases. The observed mass-velocity relationship for runaway stars (Gies \& Bolton 1986) clearly shows this trend. Hoogerwerf et al. (2001) favor a supernova scenario for $\lambda$ Cep on the basis of the difference between its age and that of the likeliest parental association. The birthplace of $\zeta$ Pup is more uncertain according to Hoogerwerf et al. (2001), but van Rensbergen et al. (1996) also favor a supernova scenario. We note however that the velocities of all three stars are well below the upper limit for the ejection of very massive stars in encounters with massive binaries (Leonard 1991). We thus consider the similarity between the estimated age of $\mathrm{BD}+43^{\circ} 3654$, and its kinematic age if it was born near the center of Cygnus OB2, as the strongest argument in support of a dynamical ejection, possibly from an original cluster containing in addition Cyg OB2 8, 9, and 22.

$\mathrm{BD}+43^{\circ} 3654$ is the first runaway star from Cygnus OB2 identified thus far, but most likely it is not the only one in such a rich association. If the fraction of runaways among O-type stars is the same for Cygnus OB2 as for the more nearby population of $\mathrm{O}$ stars, we estimate that about ten more Cygnus OB2 runaways may remain to be discovered, having the potential of providing new information on their formation environments and on the mechanisms leading to the runaway ejection.

Acknowledgements. It is as always a pleasure to acknowledge the support of the staff of the Calar Alto observatory during the execution of our 
observations. We also thank the referee, Dr. Dave van Buren, for the the detailed and constructive comments. This research has made use of the SIMBAD database operated at CDS, Strasbourg, France. It also makes use of data products from the Two Micron All Sky Survey, which is a joint project of the University of Massachusetts and the Infrared Processing and Analysis Center/California Institute of Technology, funded by the National Aeronautics and Space Administration and the National Science Foundation, as well as of data products from the Midcourse Space Experiment (MSX). Processing of the MSX data was funded by the Ballistic Missile Defense Organization with additional support from NASA Office of Space Science. This research has also made use of the NASA/ IPAC Infrared Science Archive, which is operated by the Jet Propulsion Laboratory, California Institute of Technology, under contract with the National Aeronautics and Space Administration.

\section{References}

Bate, M. R., Bonnell, I. A., \& Bromm, V. 2003, MNRAS, 336, 705 Blaauw, A. 1961, Bull. Astron. Inst. Netherlands, 15, 265

Bonnell, I. A., Larson, R. B., \& Zinnecker, H. 2007, in Protostars and Planets V, ed. B. Reipurth, D. Jewitt, K. Keil (Univ. of Arizona Press)

Bouret, J.-C., Lanz, T., Hillier, D. J. 2005, A\&A, 438, 301

Comerón, F., \& Torra, J. 1994, ApJ, 423, 652

Comerón, F., \& Kaper, L. 1998, A\&A, 338, 273

Comerón, F., Torra, J., \& Gómez, A. E. 1998, A\&A, 330, 975

Comerón, F., Pasquali, A., Rodighiero, G., et al. 2002, A\&A, 389, 874

de Donder, E., Vanbeveren, D., van Bever, J. 1997, A\&A, 318, 812

Dickey, J. M., \& Lockman, F. J. 1990, ARA\&A, 28, 215

Elmegreen, B. G. 1998, in Origins, ed. C. E. Woodward, J. M. Shull, \& H. A. Thronson, ASP Conf. Ser., 148

Garmany, C. D. 1980, Conti, P. S., \& Massey, P. 1982, ApJ, 242, 1063

Gies, D. R., \& Bolton, C. T., 1986, ApJS, 61, 419

Hanson, M. M. 2003, ApJ, 597, 957

Hardorp, J., Theile, I., \& Voigt, H. H. 1964, Luminous Stars in the Northern Milky Way (LS), Vol. 3., Hamburger Sternwarte and Warner \& Swasey Obs Heap, S. R., Lanz, T., \& Hubeny, I. 2006, ApJ, 638, 409

Heger, A., \& Langer, N. 2000, ApJ, 544, 1016

Hoogerwerf, R., de Bruijne, J. H. J., \& de Zeeuw, P. T. 2000, ApJ, 544, L133

Hoogerwerf, R., de Bruijne, J. H. J., \& de Zeeuw, P. T. 2001, A\&A, 365, 49
Kiminki, D. C., Kobulnicky, H. A., Kinemuchi, K., et al. 2007, ApJ, in press Knödlseder, J. 2000, A\&A, 360, 539

Knödlseder, J. 2003, in A Massive Star Odyssey: From Main Sequence to Supernova, ed. K. van der Hucht, A. Herrero, \& C. Esteban, ASP Conf. Ser. Langer, N., Heger, A., \& Fliegner, J. 1998, in Fundamental Stellar Properties: The Interaction between Observation and Theory, IAU Symp. 189, ed. T. R. Bedding, A. J. Booth, J. Davis (Kluwer Acad. Publ.)

Leonard, P. J. T. 1991, AJ, 101, 562

Leonard, P. J. T., \& Duncan, M. J. 1988, AJ, 96, 222

Leonard, P. J. T., \& Duncan, M. J. 1990, AJ, 99, 608

Maíz Apellániz, J., Walborn, N. R., Galué, H.Á., \& Wei, L. H. 2004, ApJS, 151, 103

Markova, N., Puls, J., Repolust, T., \& Markov, H. 2004, A\&A, 413, 693

Martins, F., Schaerer, D., \& Hillier, D. J. 2005, A\&A, 436, 1049

Massey, P., \& Thompson, A. B. 1991, AJ, 101, 1408

Meynet, G., \& Maeder, A. 1997, A\&A, 321, 465

Meynet, G., \& Maeder, A. 2000, AR\&A, 38, 143.

Meynet, G., Maeder, A., Schaller, D., \& Charbonnel, C. 1994, A\&AS, 103, 97

Noriega-Crespo, A., van Buren, D., \& Dgani, R. 1997, AJ, 113, 780

Poveda, A., Ruiz, J., \& Allen, C. 1967, Bol. Obs. Tonantzintla y Tacubaya, 4, 86 Preibisch, T., Balega, Y., Hofman, K.-H., Weigelt, G., Zinnecker, H. 1999, New Astr., 4, 531

Price, S. D., Egan, M. P., Carey, S. J., Mizuno, D. R., Kuchar, T. A. 2001, AJ, 121,2819

Repolust, T., Puls, J., \& Herrero, A. 2004, A\&A, 415, 349

Scheffler, H., \& Elsässer, H. 1987, Physics of the Galaxy and the Interstellar Medium (Springer Verlag)

Schneider, N., Simon, R., Bontemps, S., \& Motte, F. 2007, A\&A, submitted

Tokunaga, A. T. 2000, in Allen's Astrophysical Quantities, ed. A. Cox (AIP Press)

Vacca, W. D., Garmany, C. D., \& Shull, J. M. 1996, ApJ, 460, 914

Vanbeveren, D., De Loore, C., \& Van Rensbergen, W. 1998, A\&ARv, 9, 63 van Buren, D., \& McCray, R. 1988, ApJ, 329, L93

van Buren, D., Noriega-Crespo, A., \& Dgani, R. 1995, AJ, 110, 2914

van Rensbergen, W., Vanbeveren, D., \& de Loore, C. 1996, A\&A, 305, 825

Walborn, N. R., \& Fitzpatrick, E. L. 1990, PASP, 102, 379

Wilkin, F. P. 1996, ApJ, 459, L31

Zacharias, N., Monet, D. G., Levine, S. E., et al. 2004, AAS, 205, 4815 